\section{L’amusie congénitale}

Isabelle Peretz
$>$ Pour une minorité d'individus, la musique est inintelligible. Pour ces rares individus, écouter un concert classique, entendre une musique endiablée ou se laisser simplement imprégner par la musique d'un film relève du défi. Ces personnes, dites «amusiques », n'ont jamais réussi à développer ce sens musical qui caractérise l'espèce humaine. Néanmoins, ils mènent une vie normale, et peuvent même réussir des carrières exceptionnelles, comme le révolutionnaire Che Gevarra [1] ou l'économiste Milton Friedman, lauréat du prix Nobel [2]. L'aptitude musicale est donc une faculté à part. Elle peut connaître des ratés alors que toutes les autres fonctions cognitives et affectives s'épanouissent normalement. C'est pour cette raison que la condition amusique nous intéresse. Par ses échecs, l'amusique nous renseigne sur la manière dont le cerveau développe cette aptitude musicale. C'est en effet lorsqu'il est déficient que l'on appréhende au mieux le fonctionnement d'un système complexe.

Reconnue depuis plus d'un siècle [3], l'amusie congénitale a peu retenu l'attention des scientifiques. Nous avons donc recherché ces amusiques très activement, en les sélectionnnant sur des critères très sévères, de façon à être sûr que l'auto description, par les patients, de leur trouble musical correspondait bien à un trouble d'apprentissage isolé et sévère. Nous sommes parvenus à identifier et convaincre 11 adultes de participer à nos études $[4,5]$. Ces «amusiques » ont tous atteint un degré d'éducation élevé et, enfants, ont tenté en vain d'apprendre la musique. Ils ont accepté d'être soumis à un grand nombre de tests dont l'objectif était de préciser la nature de leur difficulté musicale. Ces tests, qui évaluent la maîtrise de différentes capacités musicales, comme la perception du rythme et de la mélodie, sont issus de nos travaux antérieurs menés avec des patients ayant une lésion cérébrale [6,7]. Grâce à ces recherches antérieures menées sur l'amusie dite acquise, nous disposons d'un vaste arsenal d'outils d'évaluation, qui détectent très efficacement la présence d'une anomalie.

Nous avons ainsi constaté que l'amusique a plus de difficultés à suivre la mélodie que le rythme d'une musique. À titre d'illustration, nous avons évalué la capacité de ces patients à distinguer des variations mélodiques et rythmiques dans deux séries de tests. Quelle que soit la nature de la variation, le sujet doit déterminer si deux courtes phrases musicales inédites, qui lui sont présentées successivement, sont identiques ou différentes. Une différence mélodique affectera la hauteur d'une note (dans le sens de l'aigu ou du grave, ou son appartenance à la gamme), alors qu'une différence de rythme modifiera la durée d'une note (en changeant les groupements temporels mais non la métrique). La discrimination mélodique était anormale chez tous les «amusiques ». La moitié d'entre eux souffraient aussi d'un problème rythmique, mais cette déficience rythmique ne se présentait jamais de façon isolée.

Une caractéristique de la condition amusique est la difficulté à percevoir la hauteur musicale. Cette conclusion est confirmée par les résultats obtenus lorsque le sujet effectuait d'autres tâches. Par exemple, les amusiques sont incapables de détecter une fausse note (une note hors gamme) dans une mélodie conventionnelle. Même le plus
Département de Psychologie,

Université de Montréal,

CP 6128, succursalle. Centre-

ville, Montréal H3C 3J7,

Canada.

Isabelle.Peretz@umontreal.ca

médiocre des sujets témoins (ceux-ci ayant été appariés aux patients selon l'âge, l'éducation et l'absence d'éducation musicale formelle, sans toutefois qu'ils aient une plainte particulière à l'égard de la musique) réussit mieux que n'importe quel amusique. Ainsi, l'incapacité de reconnaître une fausse note peut nous servir de test diagnostique de la condition amusique [4].

Ce problème de détection, lié à la hauteur des sons dans leur contexte musical, peut refléter une difficulté plus élémentaire. En effet, lorsque l'on fait écouter à des patients amusiques des sons successifs simples (de même durée, de même nature), sans leur imprimer de variations musicales, ils ne perçoivent pas, ou mal, une différence inférieure au demi-ton. Certains, comme Monica que nous avons étudiée de façon plus approfondie [5], ne perçoivent pas une différence de tierce (3 demi-tons). Or, notre système perceptif est équipé de façon à distinguer des écarts en hauteur bien en-deça du quart de ton. Le quart de ton est perçu correctement dès la naissance, du moins chez le bébé de moins d'un an [8]. Les amusiques souffrent donc d'un trouble de la perception sonore qui affecte la hauteur des sons. Ils n'ont aucune difficulté particulière à déceler une déviation temporelle insérée dans ces mêmes séquences de sons simples.

Cette déficience du système d'analyse de la hauteur des sons peut expliquer une série d'échecs musicaux. Notamment, elle permet d'expliquer la difficulté notoire des amusiques à reconnaître un air familier. Che Guevara, par exemple, ne reconnaissait pas l'hymne national de son pays. L'origine de cet 
échec de reconnaissance peut s'expliquer par une mauvaise distinction des variations mélodiques. En effet, la mélodie est la clé d'accès à la mémoire. Si on vous fait écouter la mélodie happy birthday après avoir supprimé toutes les variations rythmiques (ce qui revient à jouer toutes les notes avec la même durée), vous n'aurez pas de difficulté à reconnaître la chanson. En revanche, si on vous tape le rythme dans les mains, vous serez incapable de la reconnaître [9]. Ainsi, un amusique qui discerne mal la mélodie aura de la difficulté à reconnaître des airs connus même s'il parvient à en suivre le rythme. C'est effectivement le cas des 11 amusiques étudiés. Ils reconnaissent difficilement les airs populaires, à moins qu'on ne leur présente les paroles associées. Dans ce dernier cas, la reconnaissance est immédiate. Cette dissociation entre paroles et musique est révélatrice. Elle montre bien que les amusiques ont appris les chansons mais que seules les paroles ont laissé une trace dans leur mémoire.

Le trouble dont souffrent les amusiques peut donc s'expliquer par l'atteinte du système d'analyse de la hauteur des sons. Cette explication peut paraître réductionniste, vu la complexité et la multiplicité des composantes de traitement qui interviennent dans les activités musicales. Cependant, il est concevable qu'une composante essentielle du système - dans ce cas-ci, l'analyse fine des variations de la hauteur du son - soit indispensable pour permettre le développement de la faculté musicale. En d'autres termes, l'analyse fine de la hauteur des sons constituerait l'essence de la compétence musicale. Une anomalie congénitale à ce niveau nuirait au développement de la sensibilité musicale dans son ensemble. Une telle difficulté retentirait sur d'autres mécanismes essentiels à l'appréciation musicale, dont le rythme.

Cependant, la reconnaissance de la hauteur des sons est utilisée dans d'autres domaines que la seule musique, y compris dans le langage parlé. Ainsi, lorsqu'on pose la question «tu chantes maintenant? », la dernière syllabe sera prononcée en moyenne dans un registre 6 demi-tons plus aigu que le reste de la phrase. La compréhension de la question repose donc aussi sur des mécanismes de décodage de la hauteur du son. Or, les amusiques n'ont pas de difficulté à utiliser ces indices mélodiques (ou prosodiques) dans la parole. Le trouble semble donc spécifique à la musique [4]. II faut cependant remarquer que les variations en hauteur que la voix utilise sont énormes en comparaison de celles qui sont utilisées en musique. II est donc possible que l'amplitude des fluctuations inhérentes à la prosodie n'échappe pas à l'amusique, même si leur système d'analyse de la hauteur du son est défectueux. Seules les variations plus fines utilisées dans la musique mettraient en échec ce système d'analyse. Ainsi, l'origine du trouble ne serait pas l'atteinte d'une composante de traitement spécialisée pour la musique. L'atteinte concernerait une composante de traitement ordinaire, qui serait simplement plus fortement sollicitée, et donc plus développée, par l'écoute de la musique.

La question cruciale qui se pose actuellement est de savoir si l'amusie congénitale est bien une pathologie du système auditif. Pour pouvoir l'affirmer sans ambiguïté, il faut découvrir ses corrélats neuronaux. En effet, si l'anomalie du système auditif correspond à une déviance dans la formation des circuits neuronaux du cortex auditif, alors cette anomalie devrait pouvoir être révélée par l'examen du cerveau par résonance magnétique. L'identifica- tion de ces marqueurs neuronaux est au cœur de nos travaux actuels.

En conclusion, l'amusie congénitale est un échec du développement normal de la compétence musicale. Ce trouble, qui touche $4 \%$ de la population [10], s'apparente à d'autres troubles mieux connus de l'apprentissage, comme les dysphasies, les dyslexies et les dyscalculies. Ainsi, en tentant de mieux comprendre la nature et l'origine de l'amusie congénitale, nous devrions être en mesure de mieux comprendre non seulement l'organisation du cerveau qui sous-tend le sens musical, mais également les mécanismes sous-jacents aux troubles de l'apprentissage en général. ^ Congenital amusia

\section{RÉFÉRENCES}

1. Taibo II PI. Ernesto Guevara, también conocido como el Che. Buenos Aires: Planeta, 1996.

2. Friedman M. Two lucky people. Memoirs. Chicago: University of Chicago Press, 1998.

3. Grant-Allen C. Notedeafness. Mind 1878 ; 10 : 157-67.

4. Ayotte J, Peretz I, Hyde K. Congenital amusia. A group study of adults afflicted with a music-specific disorder. Brain 2002 ; 125 : 238-51.

5. Peretz I, Ayotte J, Zatorre $\mathrm{RJ}$, et al. Congenital amusia : a disorder of finegrained pitch discrimination. Neuron 2002 ; 33 : 185-91.

6. Peretz I, Kolinsky R, Tramo $M$, et al. Functional dissociations following bilateral lesions of auditory cortex. Brain 1994 ; 117 : 1283-301.

7. Liégeois-Chauvel C, Peretz I, Babaï M, Laguitton V, Chauvel P. Contribution of different cortical areas in the temporal lobes to music processing. Brain 1998 ; 121 : 1853-67.

8. Olsho L, Schoon C, Sakai R, Turpin R, Sperduto V. Auditory frequency discrimination in infancy. Dev Psychol 1982 ; 18 : 721-6.

9. Hébert S, Peretz I. Recognition of music in long-term memory : are melodic and temporal patterns equal partners? Memory Cognition 1997 ; $25: 518-33$.

10. Kalmus H, Fry D. On tune deafness (dysmelodia): frequency, development, genetics and musical background. Ann Hum Genet 1980 ; 43 : 369-82. 